Military Technical College, Kobry El-Kobbah,

Cairo, Egypt $9^{\text {th }}$ International Conference

On Aerospace Sciences \& Aviation Technology

\title{
THE EFFECT OF PARTIAL BAND INTERFERENCE ON MULTI- CARRIER DS-CDMA SYSTEMS
}

Shouman $^{\star}$ S. E., Elbarbary ${ }^{\star}$ K. A., Fareed* E. M.

\section{ABSTRACT}

In this paper, a multi-carrier signaling technique is applied to a direct sequence code division multiple access (DS-CDMA) system. This type of signaling has the desirable properties of lower chip rate, exhibiting a narrow band interference suppression effect, along with robustness to fading, without the need of either an explicit RAKE receiver or an interference suppression filter. Band limited spreading waveforms are used to prevent self-interference. The system performance over a frequency selective Rayleigh channel is evaluated analytically and through computer simulation, in the presence of partial band interference.

\section{KEY WORDS}

Spread spectrum system, Direct sequence, and Code division multiple access.

\footnotetext{
Egyptian Armed Forces.
} 


\section{I-INTRODUCTION}

Recently, there has been considerable interest in applying direct sequence spread spectrum (DS-SS) techniques to cellular communication systems. This is partly due to its robustness against fading [1], multiple access capability [2], and antiinterference capability [3], [4].

A multi-carrier DS-SS system is considered, which is capable of enhancing the system performance as it is used to combat frequency selective fading and/or narrow band interference, without requiring the use of either a RAKE structure or a notch filter [5], [6]. The receiver provides a correlator for each carrier, and the outputs from the correlators are combined with maximal ratio combiner (MRC).

An overview of the organization of this paper as follows: The following section introduces the system model for the considered multi-carrier DS-CDMA system, and section III presents the analysis of the system performance. In section IV, the performance of a multi-carrier (MC) scheme is compared with that of a single-carrier RAKE receiver in presence of narrow band interference [7]. Simulation results are presented in section V. Finally, in section VI the conclusion is presented.

\section{II-SYSTEM MODEL}

\section{II.A Transmitter Model for the MC-DS-CDMA System}

The random binary bits sequence represented by $\mathbf{d}_{\mathrm{a}}^{(\mathrm{k})}$, where " $\mathrm{a}$ " is the bit index and "k" is the user number is multiplied by a pseudo-random spreading signature sequence represented as $\mathbf{C}_{n}^{(k)}$, where " $n$ " is the chip index. It is assumed that the spreaded sequence $\left(\mathbf{d}_{\mathrm{a}}^{(\mathrm{k})} \cdot \mathbf{C}_{\mathrm{n}}^{(\mathrm{k})}\right)$ is passed through an impuise modulator, the resulting output from the impulse modulator is passed through a chip wave-shaping filter whose impulse response is $(\mathbf{h}(\mathbf{t}))$ and the output signal modulates " $\mathbf{M}$ " multiple carrier signals.. After the modulation process is done the modulated signal is transmitted through the channel. Therefore the transmitted signal of the $k^{\text {th }}$ user $\left(\mathbf{s}_{k}(t)\right)$ is given by [7]

$$
s_{k}(t)=\sum_{n=-\infty}^{\infty} d_{a}^{(k)} C_{n}^{k} h\left(t-n T_{c}+\tau_{k}\right) \cdot \sqrt{2 E_{c}} \sum_{m=1}^{M} \cos \left(w_{m} t+\theta_{k, m}\right)
$$

where $\left(\boldsymbol{\theta}_{k, m}\right)$ is a random variable uniform distributed over $[0,2 \pi]$ representing an arbitrary carrier phase, and $(\mathbf{M})$ is the number of carriers. Each user is assigned a different pseudo-random signature sequence. Also, it is assumed that $\left(\mathbf{T}_{\mathrm{b}}=\mathbf{N} \mathbf{T}_{\mathrm{c}}\right.$ ) where $\left(T_{b}\right)$ is the duration of the random binary bits sequence where $(\mathbf{N})$ is the period and $\left(T_{c}\right)$ is the duration of the pseudo-random signature sequence respectively. Fig. (1) depicts the block diagram of the transmitter model for the $k^{\text {th }}$ user

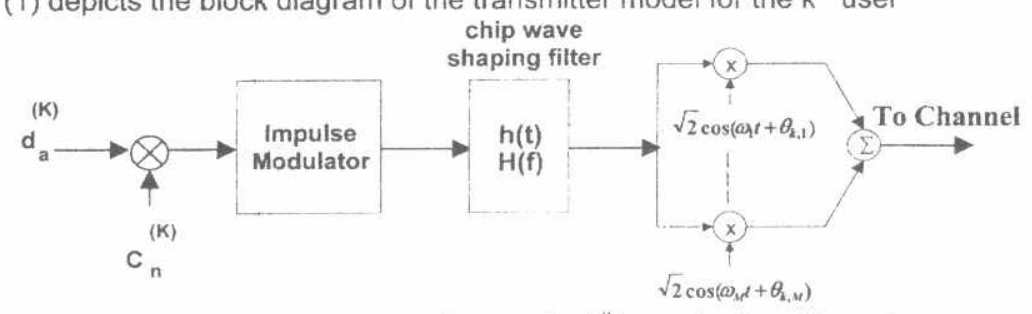

Fig. 1. The transmitter block diagram for $k^{\text {th }}$ user for the MC-system. 


\section{II.B Channel Model}

The channel is assumed to be frequency selective with Rayleigh fading and time delay spread of " $T_{m}$ " [8]. The number of carriers "M" in the MC-DS-CDMA system must be chosen to meet the following conditions: -

- Each sub - band of a MC-system has no selectivity, i.e., $T_{m} /\left(M T_{c}\right) \leq 1$.

- All sub-bands are subject to independent fading, i.e., $B W_{m c} \geq(\Delta f)_{c}$ Where $\left(\mathbf{B W}_{\mathrm{mc}}\right)$ is the bandwidth of the $\mathrm{MC}$-system, where $\left(\mathbf{B W}_{\mathrm{mc}}\right)$ can be defined as:

$$
\mathrm{BW}_{\mathrm{me}}=\frac{\mathrm{BW}_{\mathrm{sc}}}{\mathrm{M}}=(1+\beta) \frac{1}{\mathrm{MT}_{\mathrm{c}}}
$$

$(\Delta f)_{c}$ is the coherence bandwidth of the channel.

$$
\frac{T_{m}}{T_{c}} \leq M \leq(1+\beta) \frac{T_{m}}{T_{c}}
$$

Under the above assumptions, the transfer function of the $\mathrm{i}^{\text {th }}$ frequency band can be modeled for the $k^{\text {th }}$ user as:

$$
\widetilde{\zeta}_{k, i} \equiv \alpha_{k, i} \exp \left(j \lambda_{k, i}\right)
$$

where $\left(\boldsymbol{\alpha}_{\mathbf{k}, \mathrm{i}}\right)$ is an independent and identically distributed Rayleigh random variable with a unit second moment and $\left(\lambda_{\mathbf{k}, \mathbf{i}}\right)$ is an independent and identically distributed uniform random variable over $[0,2 \pi]$.

\section{II.C Receiver Model}

The input signal to the MC-DS-CDMA system $(r(t))$ can be written in the form:

$$
\begin{aligned}
r(t)= & \sum_{k=1}^{K}\left\{\sqrt{2 E_{c}} \sum_{n=-\infty}^{\infty} d_{a}^{(k)} C_{n}^{(k)} h\left(t-n M T_{c}-\tau_{k}\right) \sum_{m=1}^{M} \alpha_{k, m} \cos \left(w_{m} t+\theta_{k, m}^{\prime}\right)\right\} \\
& +n_{w}(t)+n_{J}(t)
\end{aligned}
$$

where $\left(E_{c}\right)$ is the energy per chip of the pseudo-random spreading signature sequence, $\mathbf{a}$ is the bit index, which can be defined as $\mathbf{a}=\left\lfloor\frac{\mathbf{n}}{\mathbf{N}}\right\rfloor,\left(\tau_{\mathrm{k}}\right)$ is an arbitrary time delay, $\theta_{k, m}^{\prime}=\theta_{k, m}+\psi_{k, m},\left(n_{w}(t)\right)$ is the additive white Gaussian noise "AWGN" with a double-sided power spectral density "psd" of " $\eta_{0} / 2$ ", and $\left(n_{J}(t)\right)$ is the partial band Gaussian interference with a psd of $\left(S_{n_{j}}(f)\right)$, which can be defined as:

$$
\mathrm{S}_{\mathrm{n}_{\mathrm{J}}}(\mathbf{f})=\left\{\begin{array}{lc}
\frac{\eta_{\mathrm{J}}}{2}, & \mathbf{f}_{\mathrm{J}}-\frac{\mathbf{W}_{\mathrm{J}}}{2} \leq|\mathbf{f}| \leq \mathbf{f}_{\mathrm{J}}+\frac{\mathrm{W}_{\mathrm{J}}}{2} \\
0, & \text { elsewhere }
\end{array}\right.
$$

where $\left(\mathbf{f}_{J}\right)$ is the center frequency of the interference signal and $\left(\mathbf{W}_{J}\right)$ is the bandwidth. The frequency band occupied by the partial band interference exactly overlaps an integer number of contiguous slots. Fig. (2) depicts the Power spectral density of $\left(\mathbf{n}_{\mathrm{J}}(\mathbf{t})\right)$.

The receiver model for the MC-DS-CDMA system is shown in Fig. (3). It consists of band pass filters, carrier demodulators and code correlators. The performance of such type of receiver is evaluated, through the statistical properties of the correlator output. 
Partial band interference

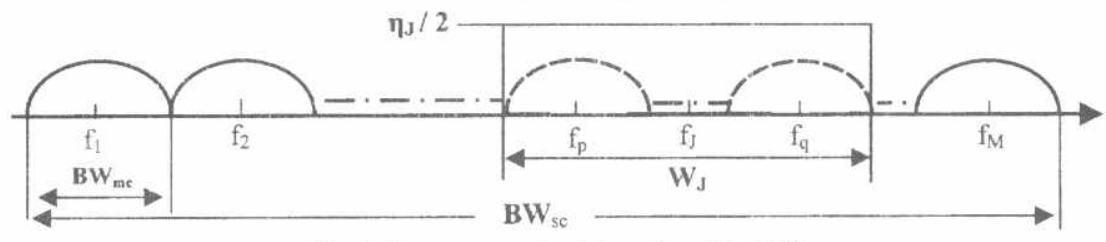

Fig.2. Power spectral density of $\left(n_{\jmath}(t)\right)$.

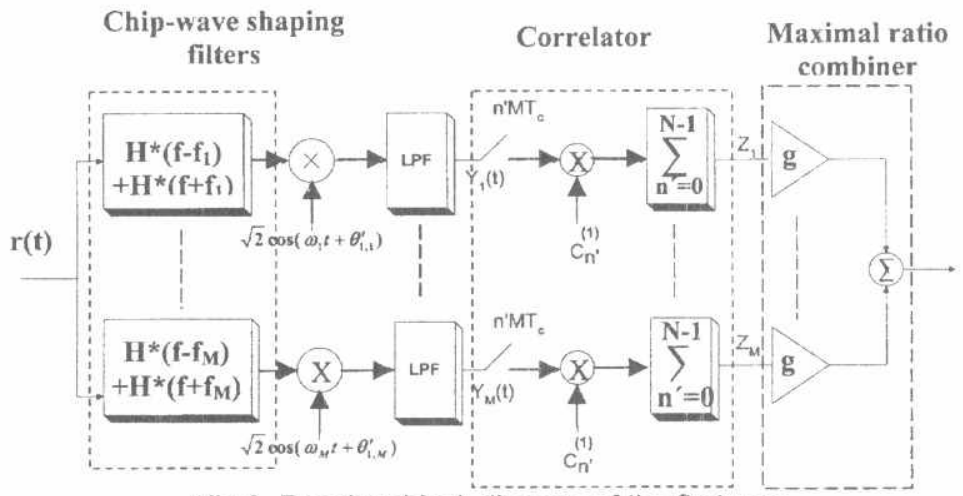

Fig.3. Receiver block diagram of the first user

\section{Analysis}

\section{III.A Correlator Output Signal}

The performance of the first user will be taken as a reference and it will be evaluated. Assuming perfect carrier, code, and bit synchronisation, the chip matched filter can be represented as, a mixer and a low pass filter, while the correlator is represented by a sampler, which samples the demodulated received bit by "QMT" samples, where $(\ell=0 \ldots N-1)$, and a decoding stage in which the sampled demodulated received signal is multiplied by a locally generated dispreading code in the receiver $\left(C_{\ell}^{(1)}\right)$, and a summator which sums the samples from $(\ell=0 \ldots \mathrm{N}-1)$. Assuming that the interference from other users, the AWGN and the partial band interference are independent of each other. The output signal from the $i^{\text {th }}$ correlator $\left(Z_{i}(t)\right)$ can be written as:

$$
\mathbf{Z}_{\mathrm{i}}=\mathrm{S}_{\mathrm{Z}_{\mathrm{i}}}+\mathbf{I}_{\mathrm{Z}_{\mathrm{i}}}+\mathbf{N}_{\mathrm{z}_{\mathrm{i}}}+\mathbf{J}_{\mathrm{Z}_{\mathrm{i}}}
$$

where, $\left(\mathbf{S}_{Z_{1}}\right)$ is the desired received data signal from the $i^{\text {th }}$ correlator,

$\left(I_{Z_{1}}\right)$ is the output from the $i^{\text {th }}$ correlator due to the interference from other users,

$\left(\mathbf{N}_{\mathrm{Z}_{1}}\right)$ is the filtered AWGN out from the $i^{\text {th }}$ correlator,

$\left(\mathbf{J}_{\mathrm{Z}_{1}}\right)$ is the output from the $i^{\text {th }}$ correlator due to the partial band interference.

The different components of each correlator output are given by: 


$$
\mathrm{S}_{\mathrm{Z}_{\mathrm{i}}}=\sum_{\ell=0}^{N-1} \mathbf{C}_{\ell}^{(1)} \cdot \mathrm{S}_{\mathrm{Y}_{\mathrm{i}}}\left(\ell \mathrm{MT}_{\mathrm{C}}\right) \text {. }
$$

where $\left(\mathbf{S}_{\mathbf{Y}_{1}}(t)\right)$ is the desired output received data signal from the $i^{\text {th }}$ chip matched filter it can be given by:

$$
S_{Y_{i}}(t)=\sqrt{E_{c}} \alpha_{1, i} \sum_{n=-\infty}^{\infty} d_{a}^{(k)} C_{n}^{(1)} x\left(t-n M T_{c}\right)
$$

where $\mathbf{X}(\mathbf{f})$ is the characteristics of the raised cosine filter representing the chip wave shaping filter.

$$
\mathbf{I}_{\mathbf{Z}_{\mathrm{i}}}=\sum_{\ell=0}^{\mathrm{N}-1} \mathbf{C}_{\ell}^{(1)} \mathbf{I}_{\mathbf{Y}_{\mathrm{i}}}\left(\ell M \mathbf{M T}_{\mathrm{e}}\right)
$$

where $\mathbf{I}_{\mathbf{Y}_{i}}(t)$ is the output from the $i^{\text {th }}$ chip matched filter due to the interference from other users, it can be written as:

$$
\mathbf{I}_{\mathbf{Y}_{\mathrm{i}}}(\mathbf{t})=\sum_{\mathrm{k}=2}^{\mathrm{K}}\left\{\sqrt{\mathbf{E}_{\mathrm{c}}} \zeta_{\mathrm{k}, \mathrm{i}} \sum_{\mathrm{n}=-\infty}^{\infty} \mathbf{d}_{\mathrm{a}}^{(\mathrm{k})} \mathbf{C}_{\mathrm{n}}^{(1)} \mathbf{x}\left(\mathbf{t}-\mathbf{n} \mathbf{M T} \mathbf{T}_{\mathrm{c}}-\tau_{\mathrm{k}}\right)\right\}
$$

where $\zeta_{\mathrm{k}, \mathrm{i}} \equiv \alpha_{\mathrm{k}, \mathrm{i}} \cos \left(\phi_{\mathrm{k}, \mathrm{i}}\right), \phi_{\mathrm{k}, \mathrm{i}}=\theta_{\mathrm{k}, \mathrm{i}}^{\prime}-\theta_{1, \mathrm{i}}^{\prime}$.

$$
\mathbf{N}_{\mathrm{Z}_{1}}=\sum_{\ell=0}^{N-1} \mathbf{C}_{\ell}^{(1)} \mathbf{N}_{\mathbf{Y}_{1}}\left(\ell \mathbf{M T}_{\mathrm{c}}\right)
$$

$\mathbf{N}_{\mathbf{Y}_{i}}(t)$ is the filtered AWGN out from the $i^{\text {th }}$ chip matched filter, it can be written as:

$$
\mathbf{N}_{\mathbf{Y}_{\mathrm{i}}}(\mathbf{t})=\mathbf{L}_{\mathrm{p}}\left\{\mathbf{n}_{\mathrm{w}, \mathrm{i}}^{\prime}(\mathbf{t}) \sqrt{2} \cos \left(\mathbf{w}_{\mathrm{i}} \mathbf{t}+\theta_{1,1}^{\prime}\right)\right\}
$$

where the term $\mathbf{n}_{w, i}^{\prime}(t)$ is the $\mathbf{n}_{w}(t)$ after passing through the $i^{\text {th }}$ band filter and the notation, and $L_{p}\{\}$ represents a low pass filtering operation and allow us to ignore the double frequency terms.

$$
\mathbf{J}_{\mathrm{Z}_{\mathrm{i}}}=\sum_{\ell=0}^{N-1} \mathbf{C}_{\ell}^{(1)} \cdot \mathbf{J}_{\mathbf{Y}_{\mathrm{i}}}\left(\ell M \mathrm{MT}_{\mathrm{c}}\right)
$$

$\mathbf{J}_{\mathbf{Y}_{\mathbf{i}}}(\mathbf{t})$ is the filtered partial band interference out from the $i^{\text {th }}$ chip-matched filter, it can be written as:

$$
J_{Y_{i}}(t)=L_{p}\left\{n_{J, i}^{\prime}(t) \sqrt{2} \cos \left(w_{i} t+\theta_{1, i}^{\prime}\right)\right\} .
$$

The term $\mathbf{n}_{\mathbf{J}, \mathbf{i}}^{\prime}(\mathbf{t})$ is the $\mathbf{n}_{\mathbf{J}}(\mathbf{t})$ after passing through the $i^{\text {th }}$ band filter.

\section{III.B Maximal Ratio Combining}

The maximal ratio combiner (MRC) combines all the output signals from the correlators in the following manner:

$$
\mathbf{Z}=\sum_{\mathbf{i}=1}^{M} \mathbf{g}_{\mathbf{i}} \mathbf{Z}_{\mathbf{i}}
$$

where $\left(g_{i}\right)$ is the gain factor of the $i^{\text {th }}$ branch. In order to maximize the signal-to-noise ratio of $\mathbf{Z}$, the branch gain factor $\left\{\mathbf{g}_{\mathbf{i}}\right\}$ is given by [7]:

$$
g_{i}=\frac{E^{2}\left\{Z_{1} \mid \alpha_{1,1}\right\}}{\operatorname{Var}\left\{Z_{1} \mid \alpha_{1, i}\right\}}
$$

The fading coefficients $\left\{\boldsymbol{\alpha}_{1,1}\right\}$ are assumed to vary sufficiently slowly so that the conditional mean and variance of $Z_{i}$ can be accurately estimated. Then the signal-tonoise ratio (SNR) $\left(\rho_{m}\right)$ can be written as: 


$$
\rho_{\mathrm{m}}=\frac{\mathbf{E}^{2}\left\{\mathbf{Z} \mid \alpha_{1}\right\}}{\operatorname{Var}\left\{\mathbf{Z} \mid \alpha_{1}\right\}}
$$

Then by using the assumption that the correlator output components are independent, it is easy to derive that:

$$
\begin{aligned}
& \rho_{\mathrm{m}}=\mathrm{N}^{2} \mathbf{E}_{\mathrm{c}} \sum_{\mathrm{i}=1}^{M} \frac{\alpha_{1, \mathrm{i}}^{2}}{\sigma_{\mathrm{I}}^{2}} \equiv \mathbf{N}^{2} \mathbf{E}_{\mathrm{c}} \gamma \\
& \text { where } \quad \sigma_{i}^{2}=\operatorname{var}\left\{\mathbf{I}_{\mathbf{z}_{1}}\right\}+\operatorname{var}\left\{\mathbf{N}_{\mathrm{z}_{1}}\right\}+\operatorname{var}\left\{\mathbf{J}_{\mathrm{z}_{1}}\right\} \\
& \gamma=\sum_{i=1}^{M} \frac{\alpha_{1, i}^{2}}{\sigma_{i}^{2}} \\
& \equiv \sum_{i=1}^{M} q_{i}
\end{aligned}
$$

Since $\left\{\boldsymbol{\alpha}_{1, i}, \mathbf{i}=1, \ldots, \mathbf{M}\right\}$ are independent and identically distributed Rayleigh random variables, $q_{i}$ has an exponential distribution with a probability density function in the form:

$$
\begin{aligned}
& f_{q}\left(q_{i}\right)=\sigma_{i}^{2} \exp \left(-\sigma_{i}^{2} q_{i}\right) \\
& \phi_{i}(s)=\frac{\sigma_{i}^{2}}{\sigma_{i}^{2}-s}
\end{aligned}
$$

where, $(\phi(s))$ is the moment generating function of $\mathbf{\gamma}$. As $q_{i}$ is an independent identically distributed random variable then the moment generating function of $\mathrm{Y}$ can be expressed as:

$$
\Phi_{\gamma}(\mathrm{s})=\prod_{i=1}^{M} \frac{\sigma_{i}{ }^{2}}{\sigma_{i}{ }^{2}-s}
$$

Finally the probability of bit error $\left(\mathbf{P}_{\mathbf{e}}\right)$ is approximated as:

$$
\begin{aligned}
& P_{e}={ }_{b}^{\infty} \phi\left(-\sqrt{N^{2} E_{c} \gamma}\right) f_{\gamma}(\gamma) d \gamma \\
& \phi(x)=\frac{1}{\sqrt{2 \pi}} \int_{-\infty}^{x} e^{-t^{2} / 2} d t
\end{aligned}
$$

$f_{y}(Y)$ is the probability density function of $\mathrm{Y}$ which is the inverse Fourier transform of $\phi(s)$.

\section{SIMULATION RESULTS}

A simulation computer program is made to compute $\mathrm{Pe}_{\mathrm{e}}$ of both the MC-system and the $S C$-system with RAKE receiver. $P_{e}$ for both systems is plotted against the SNR ranging from $(0 \mathrm{~dB}: 30 \mathrm{~dB})$ in presence of partial band interference and $A W G N$. Initially the data of different users is represented as binary phase shift keying (BPSK) which is generated randomly. The code sequences are generated by simulation of the function of maximum length feedback shift register. The jamming and the AWGN signals are simulated as band limited white Gaussian noise. The effect of the channel on the transmitted signal is represented as mentioned during the analysis of the behavior in the previous section.

The case of $\left(\mathbf{N}_{1}=511, L_{1}=4\right)$ will be considered where, $\mathbf{N}_{1}$ is the period of the pseudo random code and $L_{1}$ is the number of resolvable paths for the SC-system. The 
number of carriers $(M)$ is taken to be equal to $L_{1},(N=127, M=4)$ where, $N$ is the period of the pseudo random code for the MC-system. The curves are parameterized by the jamming to signal power ratio, (JSR) which can be defined as:

$$
\mathbf{J S R}=\frac{\eta_{\mathrm{J}} \mathbf{W}_{\mathrm{J}}}{\frac{\mathbf{E}_{\mathrm{b}}}{\mathrm{T}_{\mathrm{b}}}}
$$

where $\mathbf{E}_{\mathrm{b}}$ is the energy per bit.

Fig. (4) depicts a comparison between both systems for single user case $(\mathbf{k}=1)$ at a specific JSR, $(0 \mathrm{~dB}, 10 \mathrm{~dB}$, and $30 \mathrm{~dB})$. Assuming that the partial band jamming overlaps one slot $\left(\mathbf{W}_{\mathrm{J}}=\mathbf{B} \mathbf{W}_{\mathrm{mc}}\right)$.

One can see that, for small JSR, the performance curves of a SC-system with RAKE receiver and that of a multi-carrier system are almost the same. However, it's obvious that the MC-system outperforms the SC-system for a large JSR. This is because the MC-system is affected by the interference in only one slot. Thus, $\left(\mathbf{g}_{\mathrm{i}}\right)$ defined by equation (17) becomes very small for large interference power. Thus, the decision in the multi-carrier can be made by using the remaining uninterfered slots.

The effect of the bandwidth of the jamming signal on the behavior of the considered systems is evaluated. It is assumed that the bandwidth of the jamming signal completely overlaps the bandwidth of two and three slots at different JSR. The resultant probability of error is depicted in figures (5) and (6) for the two cases respectively. The results indicate that the behavior of both systems (MC, SC) is degraded compared to the case of only one slot jammed. However, still the MCsystem outperforms the SC-system especially for high JSR.

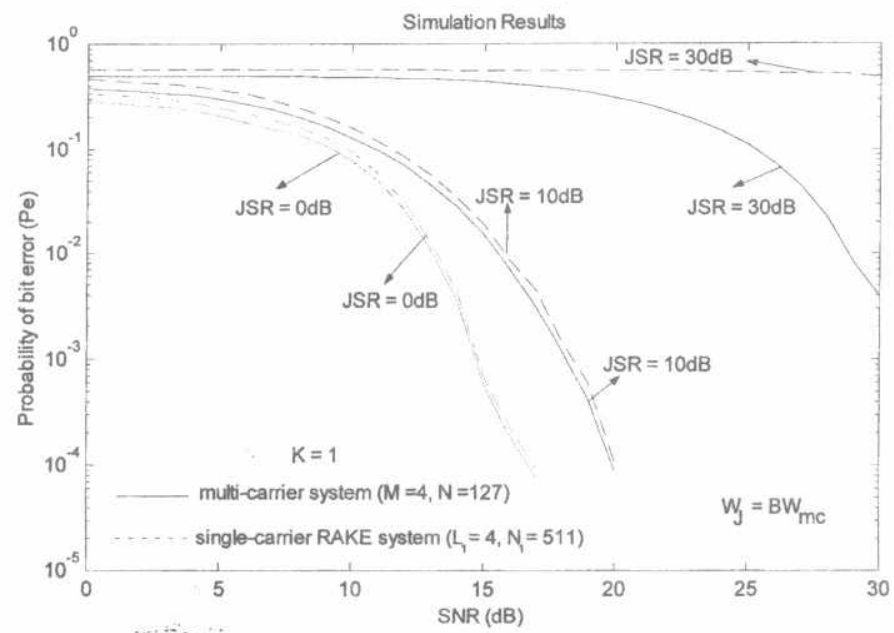

Fig.4. $P_{\mathrm{e}}$ versus $\mathrm{SNR}$ for $\mathrm{W}_{\mathrm{J}}=\mathrm{BW}_{\mathrm{mc}}$ for both systems

The effect of spreading the jamming power over a bandwidth $\mathbf{B}_{\mathfrak{J}}$, which is a variable fraction of the overall bandwidth of the MC-system is considered. The cases of one, two and three slots jammed out of the overall bandwidth of four slots are compared. 
The results are plotted in figures (7-9) for JSR $=0 \mathrm{~dB}, 10 \mathrm{~dB}$, and $30 \mathrm{Db}$ respectively. These results indicate that distribution of the total jamming power on half of the number of operating slots provides the maximum reduction in the performance of the MC-system whatever the considered JSR.

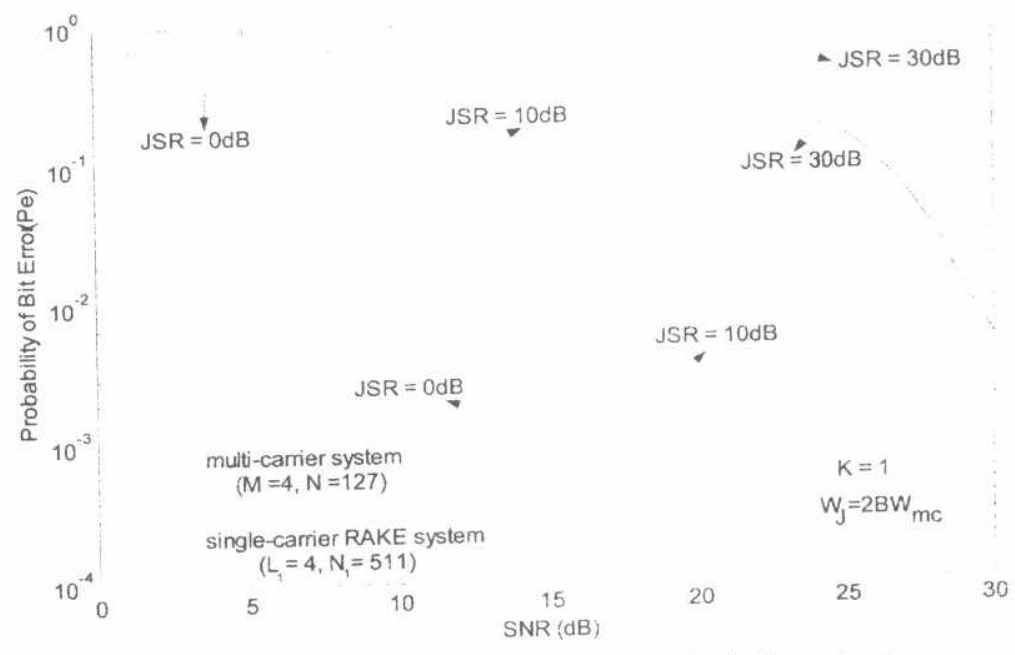

Fig.5. $P_{e}$ versus SNR for $W_{j}=2 B W_{m c}$ for both systems

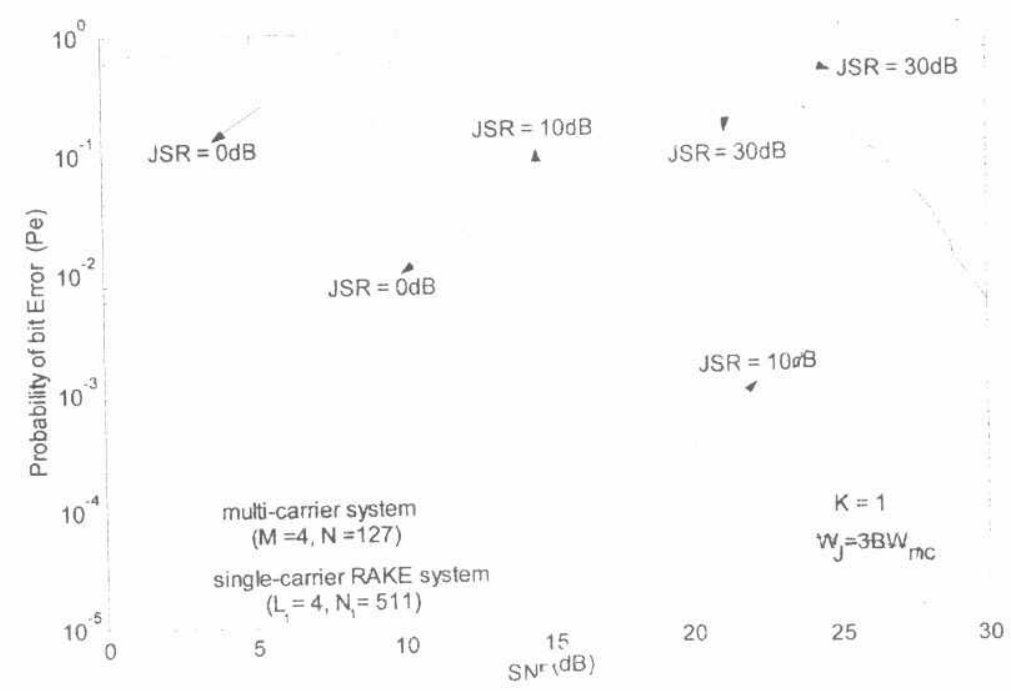

Fig.6. Pe versus SNR fr $W_{J}=3 B W m c$ for both systems 


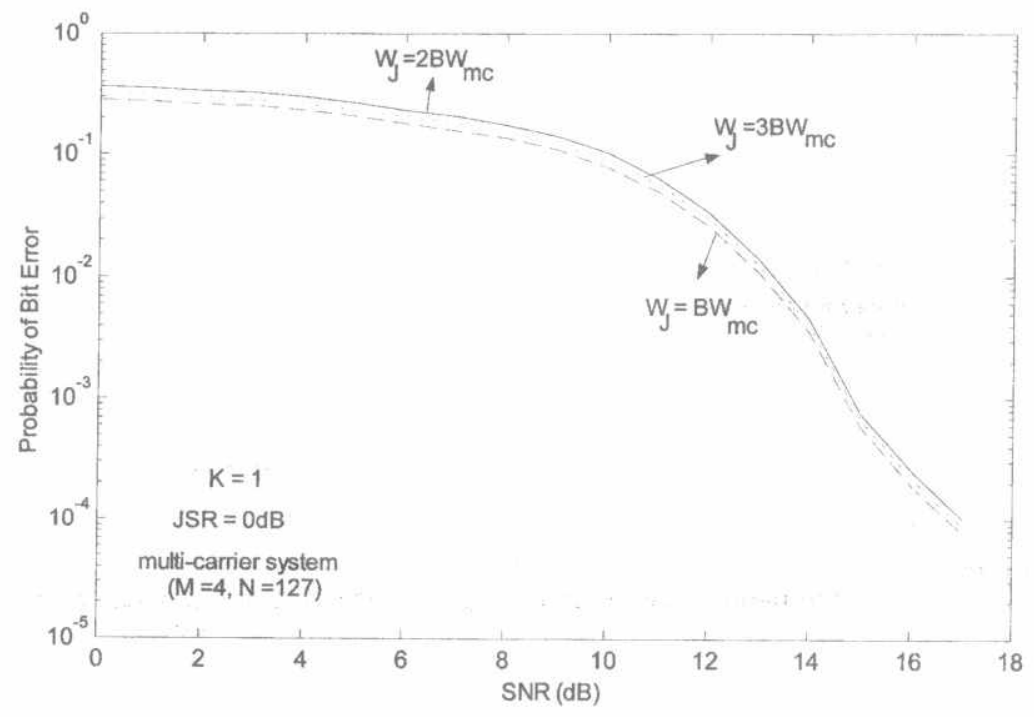

Fig.7. $P_{\mathrm{e}}$ versus $\mathrm{SNR}$ for the $\mathrm{MC}$-system at JSR=0dB

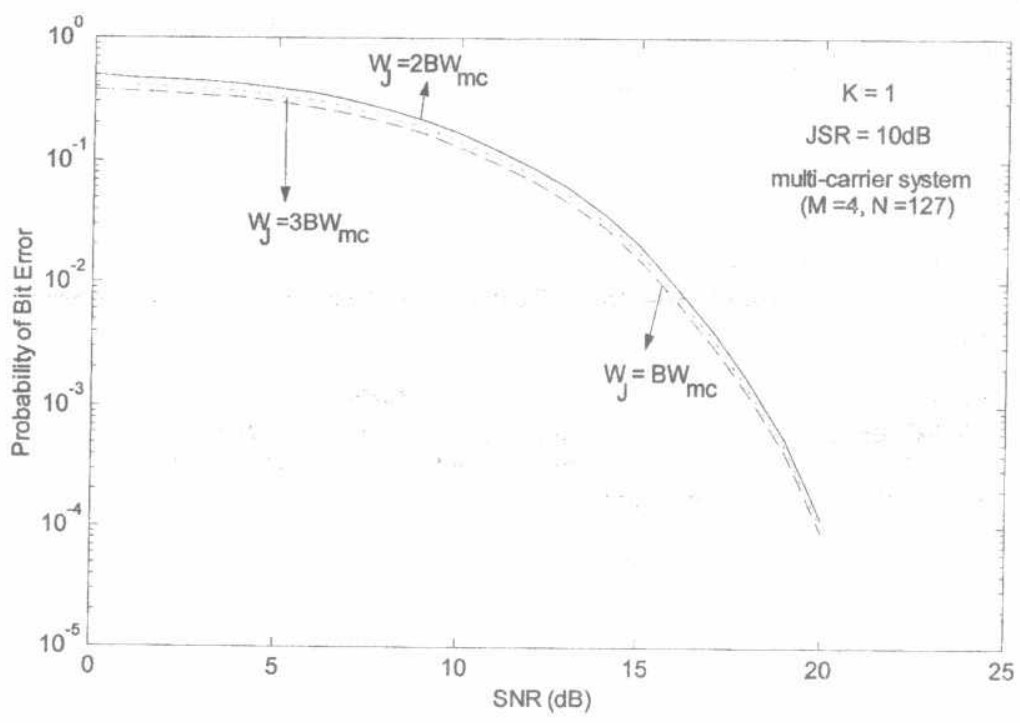

Fig. 8. $P_{e}$ versus $S N R$ for the $M C$-system at JSR=10dB 


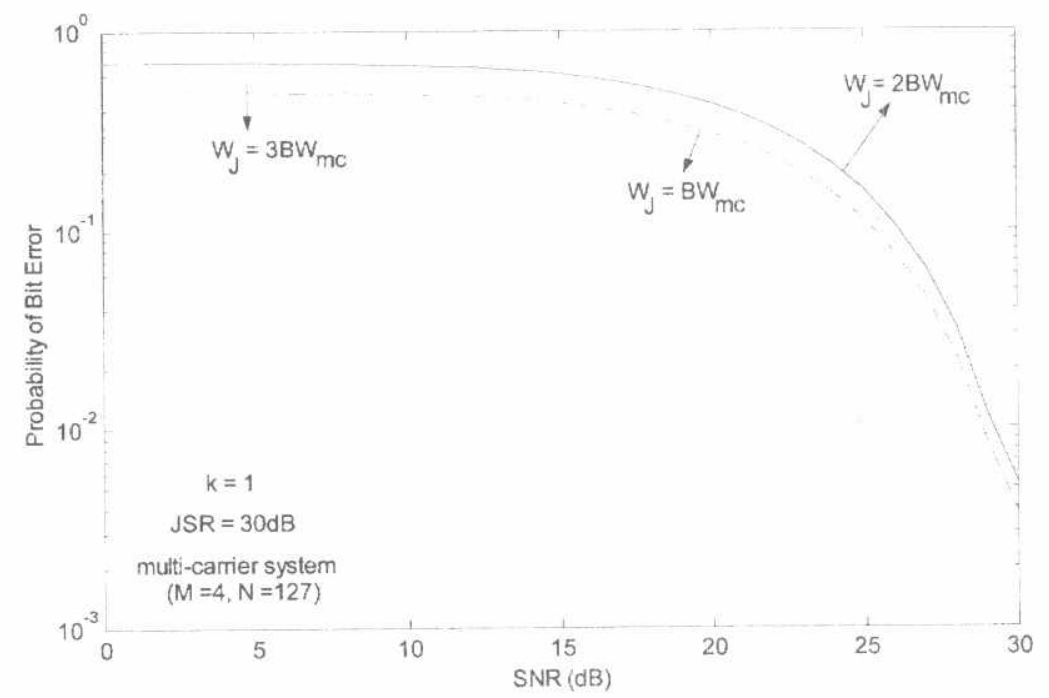

Fig.9. $P_{\mathrm{e}}$ versus SNR for the MC-system at JSR $=30 \mathrm{~dB}$

\section{CONCLUSION}

In this work, the performance of the MC-DS-CDMA system in presence of partial band interference had been analyzed and compared with the performance of the SCDS-CDMA system with RAKE receiver. The MC-system gives a better performance in presence of partial band interference than the SC-system especially for high JSR. Also, increasing the number of jammed slots degrade the performance of both systems. The maximum reduction in the performance of the MC-system will occur at a number jammed slots equal to half of the number of the operating slots whatever the considered JSR. Also, the MC-system has an advantage of using a lower chip rate. This allows the receiver to incorporate parallelized signal processing, with each of the $(\mathrm{M})$ parallel branches having a much lower computational load than that of a single serial processor for a SC-system occupying the same bandwidth. In addition, it is easier to implement the parallel receiver architecture of a number of carriers than the large order RAKE.

\section{REFERENCES}

[1] Samuel C. Yang, CDMA RF System Engineering, Artech House, Inc. (1998).

[2] R. C. Dixon, Spread Spectrum Systems, A Willy-Interscience Publication, (1975).

[3] John G. Proakis, Digital Communications, McGraw-Hill, Inc. (1983).

[4] Rahul Singh and Laurence B. Milstein, "Interference Suppression for DSCDMA", IEEE Trans. on Communications, vol. 47, no. 3, March (1999).

[5] G. L. Turin, "Introduction to Spread-Spectrum Antimultipath Techniques and their Application to Urban Digital Radio", Proc. IEEE, vol. 68, March (1980). 
[6] Kyungwhoon Cheun, "Performance of Direct-Sequence Spread-Spectrum RAKE Receiver with Random Spreading Sequences", IEEE Trans. on Communications, vol. 45, no. 9, September (1997).

[7] Shiro Kondo and Laurance B. Milstein, "Performance of Multicarrier DS CDMA Systems", IEEE Trans. on Communications, vol. 44, no. 2, February (1996)

[8] Ramjee Prasad, CDMA for Wireless Personal Communication, Artech House, Inc. (1996). 